\title{
Chemokines and Chemokine Receptors Expression in the Adipose Derived Stem Cells (ASCs), Breast Tissues and in Peripheral Blood of Patients with Breast Cancer
}

\author{
Mahboobeh Razmkhah ${ }^{1}$, Mansooreh Jaberipour ${ }^{1}$ and Abbas Ghaderi ${ }^{1,2 *}$ \\ ${ }^{1}$ Shiraz Institute for Cancer Research, Shiraz University of Medical Sciences, Shiraz, Iran \\ ${ }^{2}$ Department of Immunology, Shiraz University of Medical Sciences, Shiraz, Iran
}

\begin{abstract}
It has been demonstrated that the tumor angiogenic ability is one of the most important predictors of breas cancer progression. Factors controlling tumor angiogenesis are varied and currently under investigation. Chemokines, which produced by both tumor cells and cells of tumor microenvironment are known with a role in tumor angiogenesis. Here, we examined the expressions of SDF-1/CXCR4/CXCR7, CXCL13/CXCR5, RANTES/ CCR5, MCP-1 and CCR7 in adipose derived stem cells (ASCs) and breast cancer tissues of breast cancer patients. Results of ASCs were compared to those from sex matched healthy individuals. Data of breast cancer tissues were compared between stage III and stages I and II tumors. As a result, SDF-1 protein showed higher expression in ASCs from patients with pathological stage III compared to those with pathological stages I and II tumors and normal individuals. In breast cancer tissues, the mRNA expressions of MCP-1 and SDF-1 were 8.4 and 2.6-fold more in patients with stage III than those with stages I and II tumors. RANTES and CXCR4 mRNAs had significantly more expression in tissues of HER2 ${ }^{+}$compared to HER2 patients ( $P$ value $=0.01$ and 0.04 , respectively). Current information suggest adipose derived stem cells as one of the major players of breast cancer microenvironment which express angiogenic chemokine molecules and contribute to the breast cancer cells growth and progression.
\end{abstract}

Keywords: Breast cancer; Angiogenesis; ASCs; Chemokines; Tumor microenvironment

\section{Introduction}

Although most patients with solid tumors, including breast cancer, undergo primary surgery, they are still at considerable risk of dying from metastases to other organs [1]. During the past decade much work has been devoted to the search for predictors of tumor formation and markers of metastasis in peripheral blood. Recently, it has been demonstrated that the tumor's angiogenic capacity is one of the important features of breast cancer progression [2]. Recently, the importance of chemokines and chemokine receptors in tumorigenesis has been defined. They closely correlate with tumor cell proliferation, angiogenesis and metastasis. The stromal cell derived factor-1 (SDF-1)/CXCR4/CXCR7 axis plays important roles in the recruitment of endothelial progenitor cells, angiogenesis [3,4], and metastasis of different types of tumors [4-6]. There are several investigations explaining the expressions of chemokine and chemokine receptors in breast cancer tissues and their roles in breast cancer growth and metastasis. The most popular report linking the expressions of chemokine/chemokine receptors specially SDF-1/ CXCR4 to breast cancer metastasis has been published by Muller et al. [7]. They succeeded to inhibit the breast cancer metastasis using a CXCR4 neutralizing antibody [7]. Moreover, CXCR4 may contribute to the production of angiogenic factors such as interleukin-8 (IL-8) and vascular endothelial growth factor (VEGF) by tumor cells [8]. Through angiogenesis, regulated upon activation, normal $\mathrm{T}$ cell expressed and secreted (RANTES) and monocyte chemotactic protein (MCP)-1 has also been reported to have tumor promoting effects [9].

In contrast, the interferon-gamma-inducible protein 10 (IP-10)/ CXCR3 axis has angiostatic effects and may suppress tumor growth and progression [3]. In this connection, IP-10 activation was found to suppress the growth and recurrence of endometrial cancer [10]. In addition, the expressions of both CXCR3 and IP-10 has been shown in human breast tumors and the expression level of CXCR3 in breast tumor samples was shown to be a predictor of poor prognosis for this type of cancer [11].

It has increasingly been shown that chemokines are produced by tumor cells as well as by cells of the tumor microenvironment such as cancer-associated fibroblasts (CAFs) and mesenchymal stem cells (MSCs) [12,13]. Cancer stem cells can arise from mesenchymal stem cells (MSCs) recruited to the tumor microenvironment, as shown in the development of epithelial cancers [14]. Experiments in animal models showed that MSCs are able to migrate to tumor and injury sites after intravenous (i.v.) injection [15]. This property is strongly depends on the expressions of several chemokine receptors, especially CXCR4 on MSCs [16]. These cells may contribute to the cancer progression through several mechanisms, among which the production of proangiogenic factors such as VEGF and IL-6 [17], and also matrixdegrading enzymes such as matrix metalloproteinases (MMPs) [18] are of particular interest. Thus, MSCs might be predicted to promote, rather than inhibit, tumor growth [19]. Mesenchymal stem cells can be isolated from embryonic or adult tissues such as bone marrow and adipose tissue [20]. Adipose-derived stem cells (ASCs) have a high

*Corresponding author: Abbas Ghaderi, Shiraz Institute for Cancer Research Shiraz University of Medical Sciences, PO BOX: 71345-1798, Shiraz-Iran, Fax: 0098711230 4952; Tel: 0098711230 3687; E-mail: ghaderia@sums.ac.ir

Received June 01, 2011; Accepted September 26, 2011; Published September 29, 2011

Citation: Razmkhah M, Jaberipour M, Ghaderi A (2011) Chemokines and Chemokine Receptors Expression in the Adipose Derived Stem Cells (ASCs), Breast Tissues and in Peripheral Blood of Patients with Breast Cancer. J Carcinogene Mutagene 2:120. doi:10.4172/2157-2518.1000120

Copyright: (c) 2011 Razmkhah M, et al. This is an open-access article distributed under the terms of the Creative Commons Attribution License, which permits unrestricted use, distribution, and reproduction in any medium, provided the original author and source are credited. 
proliferative capacity, and can enter the tumor vessels and differentiate into endothelial cells. Muehlberg and colleagues demonstrated that ASCs can home to the tumor microenvironment and promote breast tumor formation and growth [21].

Based on these reports, this study focused on the expressions of several chemokine and chemokine receptors in ASCs isolated from adipose tissue of breast, breast cancer cells and peripheral blood of breast cancer patients.

\section{Materials and Methods}

\section{Subjects}

Sixty women diagnosed with breast cancer (90\% with infiltrating ductal carcinoma and $10 \%$ with the other types of breast cancer) referred to our laboratory from the Breast Clinic of the Shiraz University of Medical Sciences, Shiraz, Iran during the years 2008 to 2010. This group was examined for the expressions of SDF-1, CXCR4, CXCR7, IP-10, and CXCR3 mRNAs in their peripheral blood. Among this group, twenty patients were chosen for isolating ASCs and breast cancer cells in order to determine the expression of SDF-1/CXCR4/ CXCR7, CXCL13/CXCR5, RANTES/CCR5, MCP-1 and CCR7. None of the patients received radiotherapy, chemotherapy or immunotherapy before sampling. Data of peripheral blood were compared to those from forty sex matched healthy individuals and data from ASCs were compared to those extracted from 15 sex matched healthy volunteers with no history of malignancy or autoimmune disorders. The mean age of the patients was 51 years, whereas healthy controls were aged 55 years. Data of breast cancer tissues were compared between patients with stage III and those with stages I and II. Among those patients recruited for isolation of ASCs and tissues, there were 2 patients with pathological stage I, 11 with pathological stage II and 7 with pathological stage III. All participants have given informed consent to take part in the study.

\section{ASCs isolation and culture}

ASCs were isolated and cultured as previously described [22]. Briefly, fragments of adipose tissue isolated from breast tissue of breast cancer patients and normal individuals from mamoplasty surgery, were washed with phosphate buffered saline (PBS), minced in small pieces and digested with $0.2 \%$ collagenase at $37^{\circ} \mathrm{C}$ on a shaker for 2 hours. The resulted soup was centrifuged at $400 \mathrm{~g}$ for 10 minutes. Then the pellet including the adherent stromal cells was carefully put on Ficoll (Biosera, UK) and centrifuged at $400 \mathrm{~g}$ for 30 minutes. The second white layer, stromal vascular fraction (SVF), was transferred into a tube and washed with PBS. The SVF pellet was resuspended in DMEM culture medium (GIBCO, USA) containing $10 \%$ fetal bovine serum (GIBCO, USA) and 1\% penicillin/streptomycin (Biosera, UK). Non-adherent cells were discarded after $24 \mathrm{hrs}$ culturing. Adherent cells were cultured by changing medium every 4 days and harvested in passage 3 nearly after 30 days culture.

\section{Flow cytometry analysis}

As shown previously [22], ASCs were harvested by treatment with $1 \%$ trypsin-EDTA (Biosera, UK). $5 \times 10^{6}$ of trypsinized cells were washed twice with PBS and stained with fluorescein isothiocyanate (FITC)-conjugated mouse anti-human CD14, CD34, CD45 and CCR5 (BD Biosciences) and phycoerythrin (PE)-conjugated mouse antihuman CD44, CD105 and CD166 (BD Biosciences). For intracellular staining of RANTES, IP-10 and MCP-1, ASCs were incubated with Brefeldin $\left(1 \mu \mathrm{l}\right.$ per $\left.1 \times 10^{6} \mathrm{ASCs}\right)$ for 5 hours. After incubation time, cells were fixed and permeabilized with $0.2 \%$ saponin then stained with $5 \mu \mathrm{l}$ PE-conjugated mouse anti-human IP-10 antibody (BD Biosciences). Cells were also stained with FITC- or PE-labeled mouse isotype control antibodies as negative controls. After 30 minutes incubation at room temperature, cells were washed twice with PBS. Approximately 20,000 events were collected and further analyzed with the use of WinMDI 2.5 software.

\section{ASCs differentiation to chondrocytes}

As shown in our previous work [23] to show the stem cell characteristics of ASCs, they were differentiated into chondrocytes. Briefly, $1 \times 10^{5}$ passage 3 ASCs were cultured in 24 well tissue culture plate and used for chondrogenic differentiation. When ASCs were confluent to $60-80 \%$, they were used for chondrogenic differentiation using chondrogenesis differentiation kit (STEMPRO Chondrogenesis Differentiation Kit, GIBCO, USA) and stained with $0.2 \%$ Safranin O (Merck, Germany) in PBS after 2 weeks. Using Q-PCR method, chondrogenic differentiation was assessed on days 7 and 14 for mRNA expressions of collagen type II, SOX9 and aggrecan.

\section{RNA extraction and cDNA synthesis}

Approximately $1 \times 10^{6}$ passage 3 ASCs were trypsinized, washed with PBS and dissolved in $150 \mu \mathrm{l}$ ice-cold PBS and used for RNA extraction using TRIzol Reagent (Invitrogen, Germany) and Chloroform (Merck, Germany). Breast cancer tissues isolated from breast cancer patients were cut into the small pieces and more minced on ice using a homogenizer device (Hettich, Germany). Total RNA was extracted using TRIzol Reagent (Invitrogen, Germany) and Chloroform (Merck, Germany). Moreover, $1 \mathrm{ml}$ peripheral blood with EDTA was collected and promptly sent to the laboratory for isolation of total RNA. Briefly, $10 \mathrm{ml} \mathrm{RBC}$ lysis buffer was added to $1 \mathrm{ml}$ peripheral blood and tubes were centrifuged at $600 \mathrm{xg}(1400 \mathrm{rpm})$ for 10 minutes in a $4{ }^{\circ} \mathrm{C}$ microcentrifuge. Supernatant was discarded, the cell pellet gently resuspended in RBC lysis buffer again and centrifuged at $4^{\circ} \mathrm{C}$ at $3000 \mathrm{rpm}$ for 2 minutes. This step was done one more time in order to remove all remain RBCs. The resulted cell pellet was dissolved in $150 \mu \mathrm{l}$ ice-cold PBS, and used for RNA extraction using $1 \mathrm{ml}$ TRIzol Reagent (Invitrogen, Germany) and $300 \mu \mathrm{l}$ Chloroform. cDNA was produced from the extracted RNAs using the cDNA synthesis kit based on the manuscript (Fermentas, Canada).

\section{Quantitative Real Time Polymerase Chain Reaction (Q-PCR)}

For Q-PCR method, PCR reactions were carried out in duplicate for each sample and the mean of $C_{T}$ s were used to calculate $2^{-\Delta C}{ }_{T}$. Approximately $2 \mu \mathrm{l}$ of cDNA was amplified in each $25 \mu \mathrm{l}$ PCR reaction mix containing $12.5 \mu \mathrm{l}$ of $2 \mathrm{x}$ SYBR Green Master Mix (Fermentas, Canada), $0.2 \mu \mathrm{l}$ of each $10 \mathrm{pmol}$ forward and reverse primers (designed in primer 3 software, Table 1) and 10.1 $\mu$ DEPC water. PCR amplification was done in 40 cycles using the following program: $95^{\circ} \mathrm{C}$ for 10 minutes, $95^{\circ} \mathrm{C}$ for $15 \mathrm{~s}, 5^{\circ} \mathrm{C}$ for $30 \mathrm{~s}$ and $60^{\circ} \mathrm{C}$ for $34 \mathrm{~s}$. In all cases data were compared to the beta actin housekeeping gene.

\section{Western blot}

Proteins were extracted from ASCs using the RIPA buffer (MILLIPORE, USA), $10 \mu \mathrm{l}$ PMSF (Fluka, USA) and $10 \mu \mathrm{l}$ protease inhibitor Cocktail (Sigma, Germany). Samples $(70 \mu \mathrm{g}$ protein per lane) were run on SDS-PAGE gel for 90 minutes at $100 \mathrm{~V}$ and then transferred to nitrocellulose membrane. Blots were blocked in $5 \%$ nonfat skim milk in PBS with $0.1 \%$ Tween 80 for 16 hrs at $4{ }^{\circ} \mathrm{C}$. After 4 times washing with wash buffer ( $0.1 \%$ Tween 80 in PBS), blots were 


\begin{tabular}{|l|l|l|}
\hline \multirow{2}{*}{ Gene } & Sequences & Forward \\
\cline { 2 - 3 } & Reverse & \\
\hline Beta actin & CACCATCACGCCCTGGTGCC & ACAGAGCCTCGCCTTTGCCG \\
\hline SDF-1 & CAGCCGGGCTACAATCTGAA & TGCCAGAGCCAACGTCAAG \\
\hline IP-10 & CAAAATTGGTTGCAGGAAT & AGGAACCTCCAGTCTCAGCA \\
\hline CXCL13 & ACCATTCCCACGGGGCAAGA & GCCTCTCTCCAGTCCAAGGTGT \\
\hline RANTES & CTCAAGTGATCCACCCACCT & GAGGCTTCCCCTCACTATCC \\
\hline MCP-1 & TGGAATCCTGAACCACTTC & CCCCAGTCACCRGCTGTTAT \\
\hline CXCR4 & TGGAGTGTGACAGCTTGGAG & GGTGGTCTATGTTGGCGTCT \\
\hline CXCR7 & AAGGCCTTCATCAGCTCGTA & ACGTGGTGGTCTTCCTTGTC \\
\hline CXCR3 & CAGTCACTGCTGAGCTGGAG & AGCTCTGAGGACTGCACCAT \\
\hline CXCR5 & TGAAGGAGGCCATGAGGGGC & ACCTGGAGGACCTGTTCTGGG \\
\hline CCR5 & AACCTTCTGCAACACCAACC & GGCAAAGACAGAAGCCTCAC \\
\hline CCR7 & TGTAGGGCAGCTGGAAGACT & GATGCGATGCTCTCATCA \\
\hline
\end{tabular}

Table 1: The sequences of primers used for detecting the mRNA expressions of beta catin, chemokines and chemokine receptors.

then incubated for $1 \mathrm{hr}$ either with a rabbit anti-CXCR4 antibody (Abcam, Cambridge, MA), a rabbit anti-SDF-1 (Abcam, Cambridge, MA) or a mouse anti- $\beta$-actin antibody (Abcam, Cambridge, MA). Blots were then washed four times with wash buffer and incubated for 1:30 hrs at room temperature with a horseradish peroxidase-conjugated anti-rabbit or anti-mouse secondary antibody (Abcam, Cambridge, MA). Finally, blots were washed four more times with wash buffer and observed via enzyme-linked chemiluminescence using the SuperSignal West Pico Chemiluminescence kit (Pierce, USA).

\section{Statistical analysis}

mRNA expressions of described genes were determined using $2^{-\Delta C_{T}}$ method. For comparison of data between patients and controls the median of $2^{-\Delta C_{T}}$ was used. Analysis of gene expression between patients and controls and with the pathological information of the patients was considered significant if $\mathrm{P}$ value $<0.05$ using the Mann-Whitney $\mathrm{U}$ test and Kruskal-Wallis $\mathrm{H}$ tests, respectively. Graphs were presented using GraphPad Prism 5 and flow cytometry data were analyzed using WinMDI 2.5 software.

\section{Results}

\section{Adipose derived stem cells}

ASCs isolation and characterization: ASCs were appeared with a spindle shape in the culture as documented in Figure 1a. They were harvested in passage 3 and characterized as mesenchymal stem cells using flow cytometry analysis. As recently determined [23], ASCs were characterized as mesenchymal stem cells through chondrogenic differentiation. Safranin O staining of differentiated cells demonstrated the morphological changes in these cells to a rounder and cuboidal shape (Figure 1b). In addition, results of Q-PCR for the expression of collagen type II, aggrecan and SOX9 proved chondrogenic
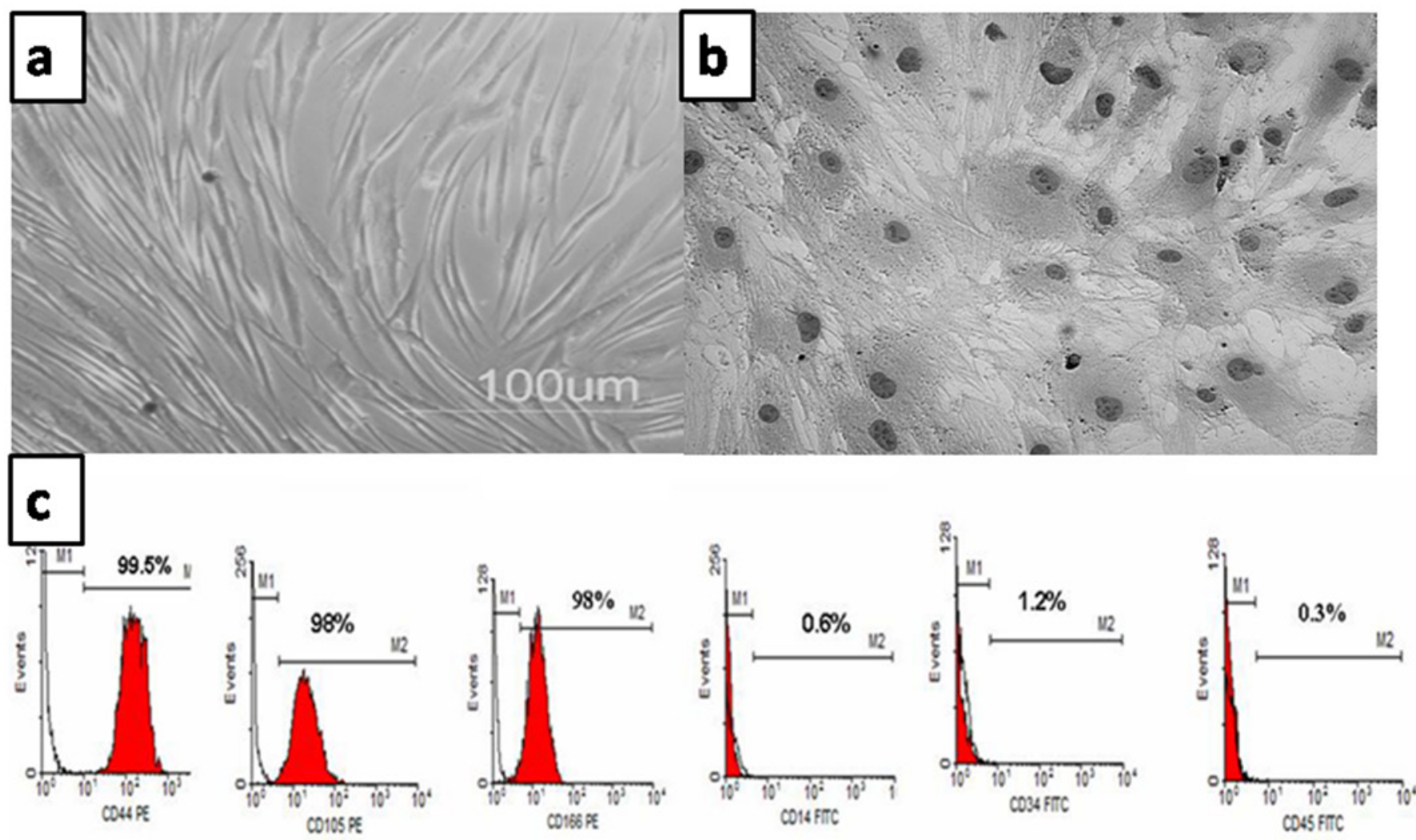

Figure 1: Adipose derived stem cells (ASCs) in culture at passage 3. They were presented with the spindle shape appearance before differentiation (a) and appeared with rounder and cuboidal shapes after chondrogenic differentiation (b) [23]. The flow cytometric analysis of ASCs showed that more than $98 \%$ of all cells were positive for the expression of CD44, CD105 and CD166. Absence of CD14, CD34 and CD45 expressions on the surface of ASCs were observed. Filled histograms represent the specific markers (CDs) and unfilled were isotype control (Figure 1c) [22]. 
differentiation of these cells [23]. As shown previously [22], flow cytometry characterization revealed that ASCs were approximately 98\% positive for the expressions of CD44, CD105 and CD166. Absence of CD14, CD34 and CD45 expressions on the surface of ASCs were observed (Figure 1c).

\section{Expressions of chemokine/chemokine receptors in ASCs}

Quantitative Real Time Polymerase Chain Reaction (Q-PCR): The median of $2^{-\mathrm{AC}}$ of different chemokine and chemokines receptors in ASCs of patients and controls have been demonstrated in Figures 2 and 3. As a result, IP-10 had 1.7-fold and CXCR4, 5-fold lower mRNA expression in patients compared to controls. In contrast, SDF1, RANTES, MCP-1, CXCL13, CXCR7 and CCR5 were expressed more in patients than controls. CXCL13 and RANTES showed 2-fold, MCP-1 1.3-fold, and CCR5 2.5-fold more expression in patients than controls. CXCR7 mRNA showed 6-fold more expression in patients than controls $(P$ value $=0.06)$. These differences were not statistically significant. There were no difference in the expressions of CXCR3, CXCR5 and CCR7 in ASCs of two groups (Figure 3).

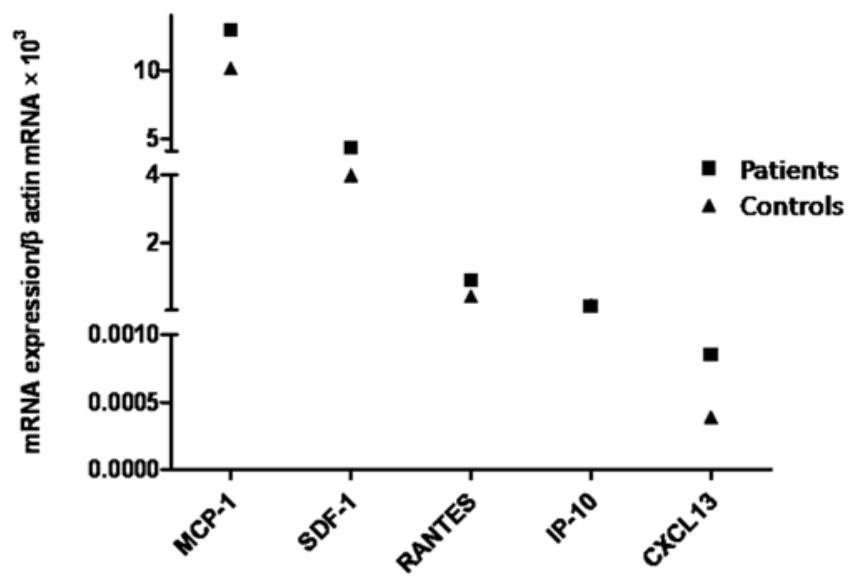

Figure 2: Expression of different chemokine mRNAs in ASCs of patients and controls. Data were shown as the median of $2^{-\Delta C} \times 10^{3}$ ( $\mathrm{P}$ value $>0.05$ for all).

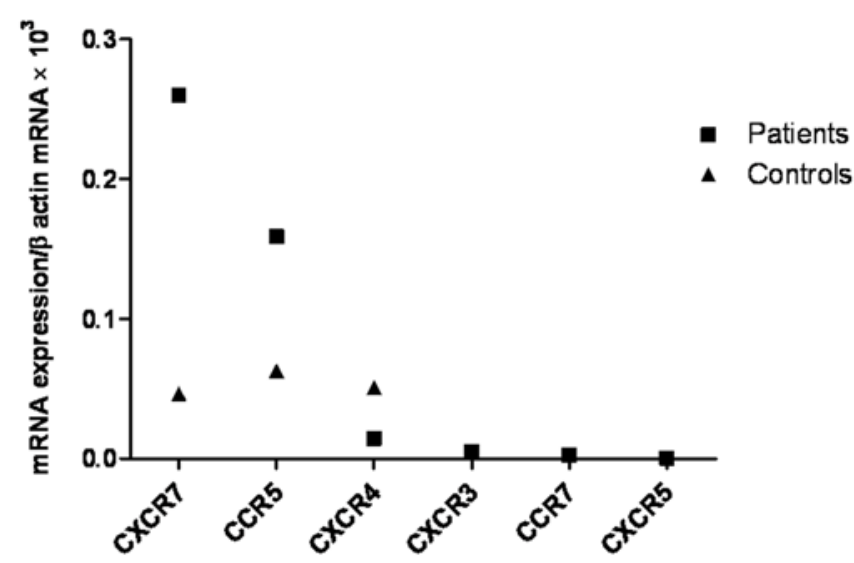

Figure 3: mRNA expression of several chemokine receptors in ASCs of patients and controls. Data were shown as the median of $2^{-\Delta C} \times 10^{3}(\mathrm{P}$ value $>0.05$ for all).

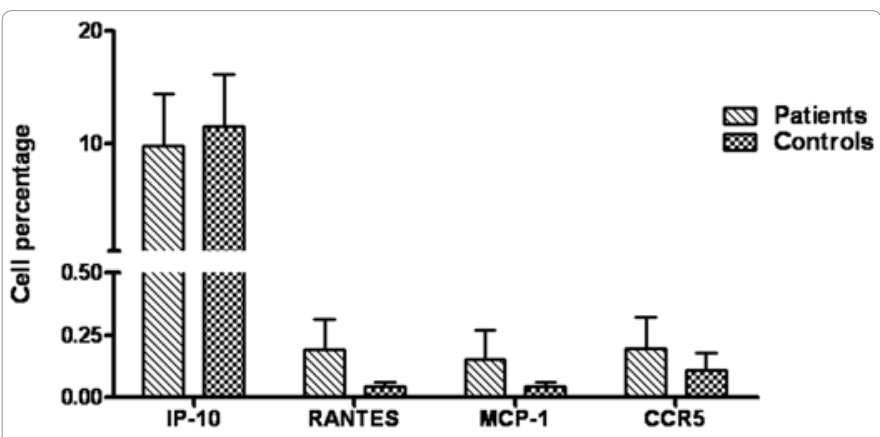

Figure 4: Flow cytometry results for the expressions of IP-10, RANTES, MCP1 and CCR5 in ASCs isolated from breast cancer patients and controls. Data were shown as mean \pm SEM of cell percentages. $P$ value $>0.05$ for all.

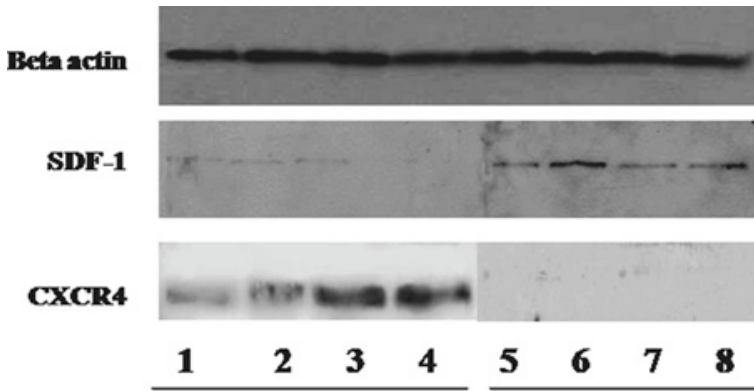

Figure 5: Results of western blot analyses of beta actin, SDF-1 and CXCR4 in protein extracts from ASCs of breast cancer patients and normal individuals. Lanes 1, 2, 3, 4: normal individuals. Lanes 5,6: ASCs of stage III patients, Lanes 7, 8: ASCs of stage II patients.

Flow cytometry for detecting IP-10, CCR5, RANTES and MCP-1: The data of Q-PCR for IP-10, CCR5, RANTES and MCP-1 expressions in ASCs was confirmed by flow cytometry. As a result, expression of IP-10 was lower in ASCs of patients than controls (Mean \pm SEM was $9.8 \pm 4.7$ in patients and $11.3 \pm 4.6$ in normal individuals). The expressions of CCR5, RANTES and MCP-1 were higher in ASCs of patients compared to those from normal individuals (Figure 4). The Mean \pm SEM for CCR5 expression was $0.2 \pm 0.1$ in patients and $0.07 \pm$ 0.03 in normal individuals. It was $0.2 \pm 0.1$ in patients and $0.04 \pm 0.02$ in normal individuals for the expressions of both RANTES and MCP-1.

Western blot for detecting SDF-1 and CXCR4 in ASCs: The data of Q-PCR for SDF-1 and CXCR4 expressions in ASCs were confirmed by western blot. As a result, SDF-1 protein was clearly expressed more in patients than normal individuals. On the other hand, patients with pathological stage III tumors significantly expressed more SDF-1 protein compared to stage II tumors as shown for two stage III, two stage II and four normal samples in Figure 5. In contrast, CXCR4 was expressed less in ASCs of patients than those from normal individuals as also indicated by Q-PCR (Figure 5).

\section{Breast cancer tissues}

SDF-1 and MCP-1 showed more mRNA expressions in stage III than stages I and II tumors. The mRNA expressions of MCP-1 and SDF-1 were 8.4 and 2.6-fold more in patients diagnosed with stage III tumors than those with stages I and II. However, these differences were not statistically significant (Figure 6). No difference was found in the 
expression of chemokines receptors in breast cancer tissues between different stages of the tumor.

Association study between the expressions of these chemokine/ chemokine receptors and clinicopathological characteristics of breast cancer patients showed that RANTES and CXCR4 mRNAs had significantly more expression in tissues of HER2 ${ }^{+}$compared to HER2 patients (P value $=0.01$ and 0.04 , respectively). CXCR7 had statistically significant more mRNA expression in tumors larger than $2 \mathrm{~cm}$ (P value $=0.05)$.

Comparison of chemokine/chemokine receptors expression between ASCs and breast cancer tissues: Compared to other chemokines, MCP-1 had the higher mRNA expression in both sites. Among chemokine receptors, CXCR7 and CXCR4 had higher expressions in ASCs and tissues, respectively. Interestingly, in ASCs CXCR7 had 26-fold higher mRNA expression compared to CXCR4, while in breast cancer tissues CXCR4 had 20-fold higher mRNA expression compared to CXCR7.

Peripheral blood: Both IP-10 and CXCR3 mRNA expression levels were significantly lower in peripheral blood of patients than controls (P value $=0.001$ and 0.05 , respectively, Figure 7). SDF-1 mRNA had lower expression in peripheral blood of patients than controls but not as lower as IP-10 $(\mathrm{P}$ value $=0.01)$. There were no significant different

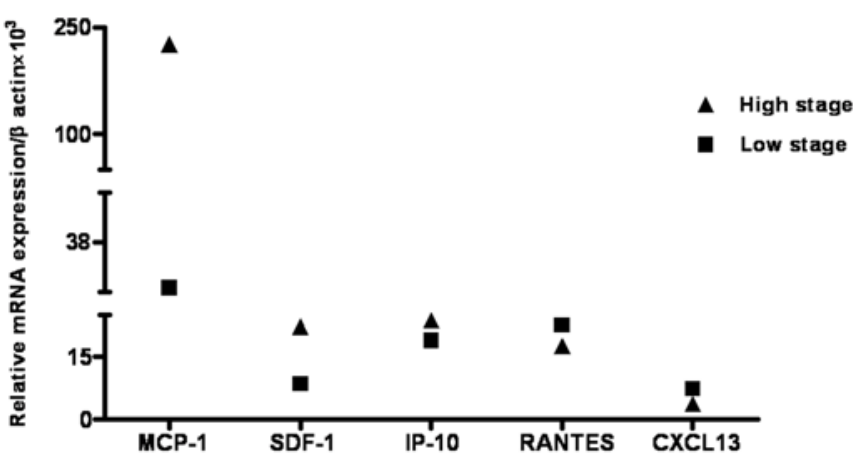

Figure 6: mRNA expressions of chemokines in patients with stage III (high stage) and stages I and II (low stage). Data were shown as the median of $2^{-\Delta C_{T}}$. $P$ value $>0.05$ for all.

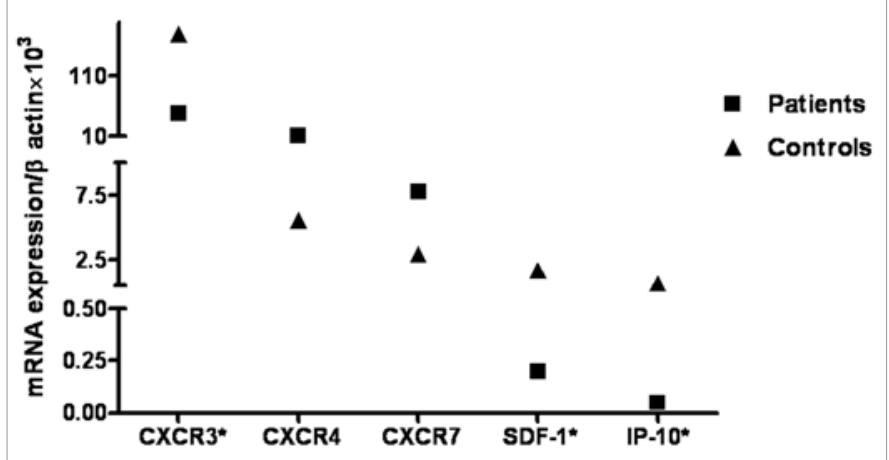

Figure 7: The comparison of Relative Quantitation (RQ) of IP-10/CXCR3 and SDF-1/CXCR4/CXCR7 mRNAs in peripheral blood of breast cancer patients and controls. Data were shown as the median of $2^{-\Delta C}$. P value $<0.05$ for IP-10, CXCR3 and SDF-1 and P value $>0.05$ for CXCR4 and CXCR7 expressions. in the expression of CXCR4 and CXCR7 mRNAs between peripheral blood of patients and normal individuals ( $\mathrm{P}$ value $>0.05$, Figure 7 ).

\section{Discussion}

Recently, role of chemokines and chemokine receptors in tumor growth and metastasis has been widely studied. Accordingly, increased production of CXCR4, CCR10 and CCR7 by breast tumor cells has been reported as important contributors to the tumor growth and metastasis to the sentinel lymph nodes [24,25]. Muller et al. [7] have documented that breast cancer cells express higher concentration of CXCR4 protein, and metastatic sites express higher levels of SDF-1 protein [7]. Moreover, a positive correlation was observed between HER2 receptor activation and increased expression of CXCR4 in breast cancer patients. Thus, it is suggested that the therapeutic effect of Herceptin (anti-HER2 antibody) may be partially mediated through down-regulation of CXCR4 activity in breast tumor cells [24]. Interestingly, secretion of a subset of cytokines such as stem cell factor (SCF) and RANTES is induced by HER2 expression in human breast cancer [26].

Our findings are in line with these reports, because SDF-1 showed 2.6-fold more mRNA expression in patients with pathological stage III than those with stages I and II tumors. Also, we found that CXCR4 and RANTES had significantly more mRNA expression in HER2 patients. Thus, based on the relationship between the expressions of HER2 and CXCR4 and higher expression of SDF-1, we conclude that SDF-1/CXCR4 production by tumor cells may influence the survival and prognosis of breast cancer patients.

In recent years, the participation of tumor microenvironment in cancer progression and development has extensively been investigated. In this case, the production of chemokine/chemokine receptors in tumor microenvironment, either by tumor cells or stromal cells such as MSCs, might be one of the most important predictors of breast cancer progression $[2,13,27]$. Since ASCs are derived from adipose tissue that is abundantly found in breast tissue, the relationship between these cells and breast cancer promotion will be attractive to be studied. It has been reported that ASCs have the ability to penetrate into tumor vessels, differentiate into endothelial cells and help to the tumor angiogenesis and invasion [21]. They may contribute to the tumor progression and metastasis by producing significant amounts of insulin-like growth factor-1 (IGF-1) [28] and some chemokines such as RANTES [29]. Furthermore, Pinilla S et al. have implicated that ASCs may contribute to the breast cancer cell invasion through the production of increased amounts of RANTES. They proposed that inhibition of invasion is possible after using anti-RANTES antibody [30].

As indicated in our previous reports breast cancer ASCs may play crucial roles for tumor development through upregulation of $\mathrm{T}$ regulatory cells [31] and the expressions of tumor promoting factors such as IL-8, VEGF, HGF and IGF-1[22]. Thus, in this study we investigated the expressions of different chemokines and chemokine receptors in ASCs of breast cancer patients and normal individuals. Our results showed that RANTES, MCP-1, CXCL13, CXCR7 and CCR5 mRNAs were expressed more in ASCs of patients than normal individuals. Although these differences between patients and controls were not statistically significant, the production of these factors by ASCs may play important roles in tumor sites in collaboration with other tumor promoting mediators such as IL-4, IL-10, IL-8, TGF- $\beta$ and growth factors. Moreover, we observed the higher expression of SDF-1 
Citation: Razmkhah M, Jaberipour M, Ghaderi A (2011) Chemokines and Chemokine Receptors Expression in the Adipose Derived Stem Cells (ASCs), Breast Tissues and in Peripheral Blood of Patients with Breast Cancer. J Carcinogene Mutagene 2:120. doi:10.4172/2157-2518.1000120

Page 6 of 7

protein by ASCs of patients with stage III tumors. These may indicate the important role of ASCs through the production of chemokine/ chemokine receptors specially SDF-1/CXCR7 in tumor sites. Muehlberg F.L. et al. have also introduced ASCs as important sources for SDF-1 in tumor site that contribute to the CXCR4 expression by tumor cells and consequently to the tumor growth, invasion and metastasis [21]. Brand and colleagues have demonstrated that ASC-derived SDF-1 stimulation induces a significant increase in proangiogenic factors such as VEGF protein levels [32]. Furthermore, it has been reported that SDF-1 may contribute to the production of other angiogenic factors such as IL- 8 and MMP-3 by tumor cells through both receptors, CXCR4 and CXCR7 $[4,8]$.

All together, results of our study may identify new outlooks for the role of both tumor cells and cells of tumor sites for cancer growth particularly through the expression of SDF-1 and its receptors. As indicated before CXCR4 was expressed more than CXCR7 in breast cancer cells while CXCR7 was expressed more in ASCs. It has been reported that the interaction of SDF-1 with CXCR4 or CXCR7 have different results due to different downstream events [11]. SDF1 interaction with CXCR4 results in chemotaxis [33], while its interaction with CXCR7 mediates proliferation [34]. Thus, SDF-1 may cause proliferation of ASCs, production of more tumor promoting mediators, and contribute to the tumor formation and growth through CXCR7 but it may lead to metastasis of tumor cells through CXCR4 in autocrine and paracrine fashions.

The results presented may illustrate the significance of chemokines, their receptors and their important sources in tumor microenvironment for tumor growth and progression. Also, the pathogenesis of cancer might not only depend on tumor cells themselves but stromal cells may also play critical roles. Thus, they may be introduced as the new therapeutic targets for breast cancer but it is required to fully characterize them before clinical applications.

\section{Conflict of interest}

The authors declare that they have no conflict of interest.

\section{References}

1. Riethdorf S, Wikman H, Pantel K (2008) Review: Biological relevance of disseminated tumor cells in cancer patients. Int J Cancer 123: 1991-2006.

2. Leek RD (2001) The prognostic role of angiogenesis in breast cancer. Anticancer Res 21: 4325-4331.

3. Vandercappellen J, Van Damme J, Struyf S (2008) The role of CXC chemokines and their receptors in cancer. Cancer Lett 267: 226-244.

4. Miao Z, Luker KE, Summers BC, Berahovich R, Bhojani MS, et al. (2007) CXCR7 (RDC1) promotes breast and lung tumor growth in vivo and is expressed on tumor-associated vasculature. Proc Natl Acad Sci U S A 104: 15735-15740.

5. Kang H, Watkins G, Parr C, Douglas-Jones A, Mansel RE, et al. (2005) Stromal cell derived factor-1: its influence on invasiveness and migration of breast cancer cells in vitro, and its association with prognosis and survival in human breast cancer. Breast Cancer Res 7: R402-410.

6. Wagner PL, Hyjek E, Vazquez MF, Meherally D, Liu YF, et al. (2009) CXCL12 and CXCR4 in adenocarcinoma of the lung: association with metastasis and survival. J Thorac Cardiovasc Surg 137: 615-621.

7. Muller A, Homey B, Soto H, Ge N, Catron D, et al. (2001) Involvement of chemokine receptors in breast cancer metastasis. Nature 410: 50-56.

8. Ping YF, Yao XH, Chen JH, Liu H, Chen DL, et al. (2007) The anti-cancer compound Nordy inhibits CXCR4-mediated production of IL-8 and VEGF by malignant human glioma cells. J Neurooncol 84: 21-29.
9. Soria G, Ben-Baruch A (2008) The inflammatory chemokines CCL2 and CCL5 in breast cancer. Cancer Lett 267: 271-285.

10. Sato E, Fujimoto J, Tamaya T (2007) Expression of interferon-gamma-inducible protein 10 related to angiogenesis in uterine endometrial cancers. Oncology 73: $246-251$

11. Fulton AM (2009) The chemokine receptors CXCR4 and CXCR3 in cancer Curr Oncol Rep 11: 125-131.

12. Lazennec G, Richmond A (2010) Chemokines and chemokine receptors: new insights into cancer-related inflammation. Trends Mol Med 16: 133-144.

13. Mishra PJ, Mishra PJ, Glod JW, Banerjee D (2009) Mesenchymal stem cells: flip side of the coin. Cancer Res 69: 1255-1258.

14. Houghton J, Stoicov C, Nomura S, Rogers AB, Carlson J, et al. (2004) Gastric cancer originating from bone marrow-derived cells. Science 306: 1568-1571.

15. Studeny M, Marini FC, Champlin RE, Zompetta C, Fidler IJ, Andreeff M (2002) Bone marrow-derived mesenchymal stem cells as vehicles for interferon-beta delivery into tumors. Cancer Res 62: 3603-3608.

16. Son BR, Marquez-Curtis LA, Kucia M, Wysoczynski M, Turner AR, et al. (2006) Migration of bone marrow and cord blood mesenchymal stem cells in vitro is regulated by stromal-derived factor-1-CXCR4 and hepatocyte growth factor-cmet axes and involves matrix metalloproteinases. Stem Cells 24: 1254-1264.

17. Kögler G, Radke TF, Lefort A, Sensken S, Fischer J, et al. (2005) Cytokine production and hematopoiesis supporting activity of cord blood-derived unrestricted somatic stem cells. Exp Hematol 33: 573-583.

18. Ries C, Egea V, Karow M, Kolb H, Jochum M, Neth P (2007) MMP-2, MT1-MMP and TIMP-2 are essential for the invasive capacity of human mesenchymal stem cells: differential regulation by inflammatory cytokines. Blood 109: 40554063.

19. Djouad F, Plence P, Bony C, Tropel P, Apparailly F, et al. (2003) Immunosuppressive effect of mesenchymal stem cells favors tumor growth in allogeneic animals. Blood 102: 3837-3844

20. Gimble JM, Katz AJ, Bunnell BA (2007) Adipose-derived stem cells for regenerative medicine. Circ Res 100: 1249-1260.

21. Muehlberg FL, Song YH, Krohn A, Pinilla SP, Droll LH, et al. (2009) Tissue-resident stem cells promote breast cancer growth and metastasis. Carcinogenesis 30: 589-597.

22. Razmkhah M, Jaberipour M, Hosseini A, Safaei A, Khalatbari B, et al. (2010) Expression profile of IL-8 and growth factors in breast cancer cells and adiposederived stem cells (ASCs) isolated from breast carcinoma. Cell Immunol 265 80-85

23. Razmkhah M, Jaberipour M, Ghaderi A (2010) Bcl-2 and Fas expressions correlate with proliferative specificity of adipose derived stem cells (ASCs) in breast cancer. Immunol Invest 40: 290-298.

24. Raman D, Baugher PJ, Thu YM, Richmond A (2007) Role of chemokines in tumor growth. Cancer Lett 256: 137-165.

25. Takeuchi H, Kitajima M, Kitagawa Y (2008) Sentinel lymph node as a target of molecular diagnosis of lymphatic micrometastasis and local immunoresponse to malignant cells. Cancer Sci 99: 441-450.

26. Jiao X, Katiyar S, Willmarth NE, Liu M, Ma X, et al. (2010) c-Jun induces mammary epithelial cellular invasion and breast cancer stem cell expansion. $J$ Biol Chem 285: 8218-8226.

27. Ma XJ, Dahiya S, Richardson E, Erlander M, Sgroi DC (2009) Gene expression profiling of the tumor microenvironment during breast cancer progression. Breast Cancer Res 11: R7.

28. Sadat S, Gehmert S, Song YH, Yen Y, Bai X, et al. (2007) The cardioprotective effect of mesenchymal stem cells is mediated by IGF-I and VEGF. Biochem Biophys Res Commun 363: 674-679.

29. Karnoub AE, Dash AB, Vo AP, Sullivan A, Brooks MW, et al. (2007) Mesenchymal stem cells within tumour stroma promote breast cancer metastasis. Nature 449: 557-563. 
Citation: Razmkhah M, Jaberipour M, Ghaderi A (2011) Chemokines and Chemokine Receptors Expression in the Adipose Derived Stem Cells (ASCs), Breast Tissues and in Peripheral Blood of Patients with Breast Cancer. J Carcinogene Mutagene 2:120. doi:10.4172/2157-2518.1000120

Page 7 of 7

30. Pinilla S, Alt E, Abdul Khalek FJ, Jotzu C, Muehlberg F, et al. (2009) Tissue resident stem cells produce CCL5 under the influence of cancer cells and thereby promote breast cancer cell invasion. Cancer Letters 284: 80-85.

31. Razmkhah M, Jaberipour M, Erfani N, Habibagahi M, Talei AR, et al. (2011) Adipose derived stem cells (ASCs) isolated from breast cancer tissue express IL-4, IL-10 and TGF- $\beta 1$ and upregulate expression of regulatory molecules on T cells: Do they protect breast cancer cells from the immune response? Cell Immunol 266:116-122.

32. Brand S, Dambacher J, Beigel F, Olszak T, Diebold J, et al. (2005) CXCR4 and CXCL12 are inversely expressed in colorectal cancer cells and modulate cancer cell migration, invasion and MMP-9 activation. Exp Cell Res 310: 117 130.

33. Bleul CC, Farzan M, Choe H, Parolin C, Clark-Lewis I, et al. (1996) The lymphocyte chemoattractant SDF-1 is a ligand for LESTR/fusin and blocks HIV1 entry. Nature 382: 829-833.

34. Burns JM, Summers BC, Wang Y, Melikian A, Berahovich R, et al. (2006) A novel chemokine receptor for SDF-1 and I-TAC involved in cell survival, cell adhesion, and tumor development. J Exp Med 203: 2201-2213. 